\title{
Squarticles as a Lipid Nanocarrier for Delivering Diphencyprone and Minoxidil to Hair Follicles and Human Dermal Papilla Cells
}

\author{
Ibrahim A. Aljuffali, ${ }^{1}$ Calvin T. Sung, ${ }^{2}$ Feng-Ming Shen, ${ }^{3,4}$ Chi-Ting Huang, ${ }^{3,5}$ and Jia-You Fang ${ }^{3,6,7,8}$
}

Received 16 August 2013; accepted 15 November 2013; published online 4 December 2013

\begin{abstract}
Delivery of diphencyprone (DPCP) and minoxidil to hair follicles and related cells is important in the treatment of alopecia. Here we report the development of "squarticles," nanoparticles formed from sebum-derived lipids such as squalene and fatty esters, for use in achieving targeted drug delivery to the follicles. Two different nanosystems, nanostructured lipid carriers (NLC) and nanoemulsions (NE), were prepared. The physicochemical properties of squarticles, including size, zeta potential, drug encapsulation efficiency, and drug release, were examined. Squarticles were compared to a free control solution with respect to skin absorption, follicular accumulation, and dermal papilla cell targeting. The particle size of the NLC type was $177 \mathrm{~nm}$; that of the NE type was $194 \mathrm{~nm}$. Approximately $80 \%$ of DPCP and $60 \%$ of minoxidil were entrapped into squarticles. An improved drug deposition in the skin was observed in the in vitro absorption test. Compared to the free control, the squarticles reduced minoxidil penetration through the skin. This may indicate a minimized absorption into systemic circulation. Follicular uptake by squarticles was 2- and 7-fold higher for DPCP and minoxidil respectively compared to the free control. Fluorescence and confocal images of the skin confirmed a great accumulation of squarticles in the follicles and the deeper skin strata. Vascular endothelial growth factor expression in dermal papilla cells was significantly upregulated after the loading of minoxidil into the squarticles. In vitro papilla cell viability and in vivo skin irritancy tests in nude mice suggested a good tolerability of squarticles to skin. Squarticles provide a promising nanocarrier for topical delivery of DPCP and minoxidil.
\end{abstract}

KEY WORDS: alopecia; diphencyprone; drug targeting; hair follicles; minoxidil; squarticles.

\section{INTRODUCTION}

Hair loss perturbs many individuals due to its association with self-esteem and its tendency to lead to depression, and dissatisfaction. Alopecia areata, found in $2 \%$ of the population, is a chronic inflammatory disorder that affects the hair follicles (1) and leads to hair loss. Topical immunotherapy utilizing diphencyprone (DPCP) recruits $\mathrm{T}$ cell subpopulations to the

$\overline{{ }^{1} \text { Department of Pharmaceutics, College of Pharmacy, King Saud }}$ University, Riyadh, Saudi Arabia.

${ }^{2}$ Department of Microbiology, Immunology, and Molecular Genetics, University of California, Los Angeles, USA.

${ }^{3}$ Pharmaceutics Laboratory, Graduate Institute of Natural Products, Chang Gung University, 259 Wen-Hwa 1st Road, Kweishan, Taoyuan 333, Taiwan.

${ }^{4}$ Chinese Herbal Medicine Research Team, Healthy Aging Research Center, Chang Gung University, Kweishan, Taoyuan, Taiwan.

${ }^{5}$ Graduate Institute of Health Industry Technology, Chang Gung University of Science and Technology, Kweishan, Taoyuan, Taiwan.

${ }^{6}$ Research Center for Industry of Human Ecology, Chang Gung University of Science and Technology, Kweishan, Taoyuan, Taiwan.

${ }^{7}$ Chinese Medicine Research and Development Center, China Medical University Hospital, Taichung, Taiwan.

${ }^{8}$ To whom correspondence should be addressed. (e-mail: fajy@mail.cgu.edu.tw) follicles in order to enhance the clearance of putative antigens so that the hairs can regrow (2). Minoxidil, another drug for treating alopecia areata, in contrast to DPCP, induces vasodilation of perifollicular vessels and proliferates dermal papilla cells (3). It is also employed as a first-line topical treatment for androgenetic alopecia approved by the US Food and Drug Administration (FDA) (4). Androgenetic alopecia is reported to affect up to $96 \%$ of Caucasian males (5).

Hair loss is generally difficult to treat. Only a $50 \%$ success rate is achieved by DPCP (6). The effect of minoxidil is impermanent because hair loss occurs 4-6 months after cessation of treatment (7). On the other hand, both drugs can induce skin irritation in some cases as a result of the employed solvents. Topically applied minoxidil is also absorbed into the systemic circulation, resulting in cardiovascular side effects (8).

An important strategy to resolve these drawbacks is through targeting the drug to the hair follicles. Encapsulating the drugs into nanoparticles may provide benefits for controlled drug release, increased drug stability, and skin targeting. Nevertheless, despite these purported advantages, the targeting of the drug to the skin or appendages by nanocarriers has so far produced disappointing clinical results (9).

The prime objective of this work was to formulate DPCP and minoxidil into nanoparticles and to assess their targeting 
ability to follicles and dermal papilla cells. Here we introduced "squarticles" for achieving this aim, a lipid nanosystem containing squalene. Lipid nanoparticles, such as solid lipid nanoparticles (SLN), nanostructured lipid carriers (NLC), and nanoemulsions (NE), are feasible as drug carriers due to their lower toxicity compared with polymeric and metallic nanoparticles (10). The main difference among these lipid nanocarriers is the composition of the inner matrix. SLN have a matrix made from crystalline solid lipids; the core of NLC is composed of mixed solid and liquid lipids; NE are nanocarriers with neat liquid lipids in their inner core (11). In the present study, squarticles were prepared in the forms of NLC and NE. Sebum is a mixture of squalene, wax esters, and glycerides (12). It is secreted by the sebaceous duct, which connects to the hair follicles. Efficient targeting into the follicles can depend on the interaction between the drug and the sebum (13). It may be expected that squalene in nanoparticles facilely fuses with sebum in the follicles.

The Franz cell was used in vitro to examine skin retention, skin penetration, and follicular accumulation of DPCP and minoxidil from nanoparticles. Qualitative approaches by fluorescence and confocal microscopies were applied to the evaluation of nanoparticle distribution in the skin and appendages. Whether minoxidil-loaded squarticles could promote the production of vascular endothelial growth factor (VEGF) in dermal papilla cells was also investigated. Finally, the possible skin irritation elicited by lipid nanoparticles was studied using both cultured papilla cells and nude mouse skin.

\section{MATERIALS AND METHODS}

\section{Materials}

DPCP, minoxidil, squalene, Pluronic F68 (PF68), and Nile red were purchased from Sigma-Aldrich (St. Louis, MO, USA). Precirol ATO 5 was obtained from Gattefossé (Gennevilliers, France). Hydrogenated soy phosphatidylcholine (SPC, Phospholipon $80 \mathrm{H} \AA$ ) was supplied by American Lecithin (Oxford, CT, USA). Cellu-Sep ${ }^{\circledR}$ T1 cellulose membrane (molecular weight cutoff of 3,500) was from Membrane Filtration Products (Seguin, TX, USA).

\section{Preparation of Squarticles}

The aqueous and lipid phases of squarticles were fabricated separately. The aqueous phase consisted of double-distilled water $(88 \%, v / v)$ and PF68 $(3.2 \%, w / v)$. The lipid phase consisted of squalene (3.5\% for NLC; $7.0 \%$ for NE), Precirol (3.5\% for NLC; none for NE), and SPC (1.8\%). Both phases were separately heated to $85^{\circ} \mathrm{C}$ for $15 \mathrm{~min}$. The aqueous phase was then added into lipid phase and mixed under homogenization at 12,000 rpm (Pro 250, Pro Scientific, Monroe, CT, USA) for $20 \mathrm{~min}$. Subsequently, a probe-type sonicator (VCX600, Sonics and Materials, Newtown, CT, USA) set at a power of $25 \mathrm{~W}$ was employed to treat the mixture for $15 \mathrm{~min}$. A $10-\mathrm{ml}$ volume was prepared for each batch. Squarticles could be divided into NLC type and NE type according to the different materials in inner cores. The concentrations of DPCP and minoxidil in the formulations were $0.15 \% \quad w / v(7.3 \mathrm{mM})$ and $0.8 \% \quad w / v(38.2 \mathrm{mM})$, respectively.

\section{Average Diameter and Zeta Potential of Squarticles}

The mean particle size and zeta potential of squarticles were assessed by a laser scattering method (Nano ZS90, Malvern, Worcestershire, UK). The nanosystems were diluted 100 -fold with double-distilled water before detection. The measurement was repeated three times each sample for three batches.

\section{Drug Encapsulation Percentages in Squarticles}

The DPCP and minoxidil encapsulation in squarticles were examined by ultracentrifugation technique (Optima MAX, Beckman Coulter, Fullerton, CA, USA). The nanoparticulate dispersions were centrifuged at $48,000 \times g$ and $4^{\circ} \mathrm{C}$ for $30 \mathrm{~min}$ for dividing the loading drugs from the free form. The supernatant and precipitate were withdrawn and analyzed by high performance liquid chromatography (HPLC).

\section{HPLC Analytical Settings}

The HPLC system was Hitachi 7-series (Tokyo, Japan). A 25-cm long LiChrospher C18 column (Merck, Darmstadt, Germany) was used as the stationary phase. The mobile phase for DPCP was acetonitrile and water (60:40). The mobile phase for minoxidil consisted of methanol and water adjusted to $\mathrm{pH} 2$ by phosphoric acid (74:26). The respective wavelengths of ultraviolet detector for DPCP and minoxidil were 295 and $281 \mathrm{~nm}$.

\section{Animals and Skin Preparation}

Female nude mice (ICR-Foxn1nu) aged 8 weeks were purchased from National Laboratory Animal Center (Taipei, Taiwan). The animal experimental protocol was reviewed and approved by Institutional Animal Care and Use Committee of Chang Gung University. All animals were housed and handled based on institutional guidelines. Full-thickness skin from the dorsal region was excised after sacrifice. To prepare the sebum-removed skin, the stratum corneum (SC) side of the skin was gently washed by cold hexane $\left(4^{\circ} \mathrm{C}\right)$ five times to remove the sebum in skin surface (14). Both intact and desebum skins were used as diffusion barriers for in vitro skin delivery experiment.

\section{In Vitro Skin Delivery}

Drug amounts in and across skin after topical delivery were measured by a Franz diffusion cell. The skin was fixed between donor and receptor compartments. Cellulose membrane was also used as diffusion barrier for release rate measurement. The donor included $0.5 \mathrm{ml}$ of drug-containing nanosystem in $88 \%$ water and $3.2 \% w / v$ PF68. A control solution (30\% propylene glycol (PG) in water) as donor vehicle was also used for comparison at an equivalent dose with nanosystems. The receptor medium consisted of $30 \%$ ethanol in $\mathrm{pH} 7.4$ buffer for maintaining the sink condition. 
The effective diffusion area between compartments was $0.785 \mathrm{~cm}^{2}$. The stirring rate and temperature were kept at $600 \mathrm{rpm}$ and $37^{\circ} \mathrm{C}$, respectively. At appropriate intervals, $300 \mu \mathrm{l}$ of receptor medium was withdrawn and immediately supplied by an equal volume of fresh medium. The samples were assayed by HPLC. Drug accumulation within the skin was measured after a 24-h delivery. The skin was removed from the Franz cell, then rinsed with water, and blotted with tissue paper. The skin sample was weighed and minced by scissors, positioned in a glass homogenizer with $1 \mathrm{ml}$ methanol, and ground for $5 \mathrm{~min}$ with an electric stirrer. The mixture was centrifuged at $10,000 \mathrm{rpm}$ for $10 \mathrm{~min}$. After filtration via a $0.45-\mu \mathrm{m}$ membrane, the sample was detected by HPLC.

\section{Drug Accumulation in Hair Follicles}

Differential stripping and cyanoacrylate skin surface coating were used to detect drug content in follicles (15). Subsequent to stripping the SC of skin removed from Franz cell, a follicular cast was prepared. A drop of superglue (ethyl cyanoacrylate 7004T, 3M, Taipei, Taiwan) was added on a glass slide, which was pressed onto the surface of SC-stripped skin. The cyanoacrylate polymerized, and the slide was expelled with one quick movement after $5 \mathrm{~min}$. The super glue remaining on the slide was scraped off and positioned in a tube with $2 \mathrm{ml}$ methanol. The tube was shaken for $3 \mathrm{~h}$. The final product was vacuumed to evaporate methanol. The mobile phase was added to dissolve the residuals for HPLC assay.

\section{VEGF Amount and Viability of Dermal Papilla Cells}

Human hair dermal papilla cells were obtained from ScienCell (Carlsbad, CA, USA). The procedures for cultivation were carried out according to a previous study (16). For the determination of VEGF, early passage (P5) cells were seeded into six-well plates at a density of $2 \times 10^{5}$ cells $/ \mathrm{ml}$. VEGF in centrifuged supernatant and cell extracts was assessed by human VEGF ELISA kit (Invitrogen, Camarillo, CA, USA). The cells were incubated for $48 \mathrm{~h}$ with minoxidil concentration at $0.01 \mathrm{M}$. The preparation method of cell supernatant and extract was the same as previous study (16). For cell viability assay, cells $(2 \times$ $10^{5}$ cells $/ \mathrm{ml}$ ) were seeded in 96-well plates for a 24-h cultivation. The nanocarriers without drug were added and incubated for $24 \mathrm{~h}$ at $37^{\circ} \mathrm{C}$. After being washed with PBS, papilla cells were incubated with $5 \mathrm{mg} / \mathrm{ml} \mathrm{3-(4,5-dimethylthiazol-2-yl)-2,5-}$ diphenyl tetrazolium bromide (MTT) in RPMI-1640 for $2 \mathrm{~h}$. Formazan crystals resulting from MTT reduction were dissolved by adding $200 \mu \mathrm{l}$ DMSO with agitation for $30 \mathrm{~min}$. The supernatant was detected by ultraviolet spectrophotometer at $550 \mathrm{~nm}$. The viability was measured as a percentage of nontreatment control.

\section{In Vivo Skin Delivery}

Nile red $(0.01 \%)$ was used as the dye in this experiment. A glass cylinder with an effective diffusion area of $0.785 \mathrm{~cm}^{2}$ was affixed to the back skin of nude mouse with glue. A 0.2$\mathrm{ml}$ nanosystems or control solution (30\% PG in water) was added to the cylinder. The application duration was $6 \mathrm{~h}$. The mouse was sacrificed and the skin was excised, washed with water. The prepared skin sample was examined by fluorescence microscopy and confocal laser scanning microscopy (CLSM).

\section{Vertical Observation of Skin by Fluorescence Microscopy}

The skin samples treated with squarticles or control solution were sectioned in a cryostat microtome at a thickness of $20 \mu \mathrm{m}$, and then mounted with glycerin and gelatin. The slices were examined with an inverted microscope (IX81, Olympus, Tokyo, Japan) using respective filter sets at 546 and $590 \mathrm{~nm}$ for excitation and emission. The skin slices were also stained by hematoxylin and eosin (H\&E) for bright-field imaging. The specimens were fixed in a $10 \%$ buffered formaldehyde solution at $\mathrm{pH}$ 7.4. Sections were dehydrated in ethanol, embedded in paraffin wax, and stained with $H \& E$.

\section{Horizontal Observation of Skin by CLSM}

The full-thickness skin was directly positioned under the lens of CLSM for observing Nile red distribution in skin. The skin thickness was scanned at $5-\mu \mathrm{m}$ increments through $Z$ axis of confocal microscope (TCS SP2, Leica, Wetzlar, Germany). The optical excitation and emission wavelengths were set to 515 and 525-605 nm, respectively. Images were taken by summing 15 fragments at various depths from skin surface.

\section{In Vivo Skin Irritation Test}

The possible skin irritancy of squarticles upon in vivo administration was analyzed after repeated application for 7 days. The test was carried out based on our previous study (17). Briefly, a 0.6-ml squarticles or control solution without drugs was pipetted on a sheet of non-woven polyethylene cloth $(1.5 \times 1.5 \mathrm{~cm})$. The cloth was topically administered onto dorsal region of nude mouse and fixed with adhesive patches. The cloth was removed and replaced with a new one each day for a period of 7 days. The treated site was measured by transepidermal water loss (TEWL, TM300, Courage and Khazaka, Köln, Germany), skin erythema (CD100, Yokogawa, Tokyo, Japan), and skin pH (PH905, Courage and Khazaka) after a 7-day treatment period.

\section{Statistical Analysis}

Statistical analysis of the differences was carried out using unpaired $t$ test and ANOVA. A 0.05 level of probability $(p<0.05)$ was taken as the level of significance. Data entry and calculation was completed by using Winks version 6 (Texasoft, Duncanville, TX, USA).

\section{RESULTS}

\section{Physicochemical Properties of Squarticles}

Table I lists the size, surface charge, and capability for entrapping drugs in squarticles. The average size of the NLC type was estimated to be $177 \mathrm{~nm}$. The NE type of squarticles demonstrated a larger size $(194 \mathrm{~nm})$ compared to the NLC 
Table I. The Characterization of the Squarticles by Particle Diameter, Polydispersity Index (PDI), Zeta Potential, and Drug Encapsulation Percentage

\begin{tabular}{lccccc}
\hline Type & Size $(\mathrm{nm})$ & PDI & Zeta potential $(\mathrm{mV})$ & DPCP encapsulation (\%) & Minoxidil encapsulation (\%) \\
\hline NLC & $177 \pm 3$ & $0.24 \pm 0.01$ & $-54.0 \pm 0.2$ & $83.7 \pm 2.1$ & $63.3 \pm 0.5$ \\
NE & $193 \pm 1$ & $0.25 \pm 0.02$ & $-56.6 \pm 1.1$ & $78.5 \pm 7.1$ & $63.5 \pm 1.1$ \\
\hline
\end{tabular}

Each value represents the mean $\pm \mathrm{SD}(n=3$ for size, PDI and zeta potential, $n=4$ for drug encapsulation)

$P D I$ polydispersity index, $N L C$ nanostructured lipid carriers, $N E$ nanoemulsions

type. The polydispersity index (PDI) was low for both systems $(\leq 0.25)$, revealing a narrow size distribution. There was no significant difference $(p>0.05)$ between the zeta potential of the NLC type $(-54 \mathrm{mV})$ and the NE type $(-57 \mathrm{mV})$. For DPCP, the encapsulation capacity of squarticles recognized by ultracentrifugation was more than $75 \%$, indicating that most of the DPCP molecules were loaded in the lipid matrix. No significant difference $(p>0.05)$ was found between the entrapment of NLC and NE. For minoxidil, loading percentages of $63 \%$ and $64 \%$ were obtained for NLC and NE, respectively.

\section{In Vitro Skin Delivery}

Delivery of DPCP and minoxidil into and through the skin was first assessed in vitro. Table II depicts skin permeation profiles of DPCP from squarticles. A 30\% PG in water was used as a free control for comparison. The DPCP deposition within skin from squarticles was higher $(p<$ $0.05)$ than that from the reference control. A varying lipid matrix changed the results of the skin deposition, with the NE type showing a greater enhancement ratio $(\mathrm{ER}=3.5)$ as compared to the NLC type $(E R=2.5)$. Determination of the penetrated drug in the receptor can indicate its presence in systemic circulation if used in vivo. DPCP flux across the skin was in the order of $\mathrm{NE}>\mathrm{NLC}>$ free control, the same as observed for recovery from the skin reservoir. Although the flux value could be changed depends upon the receptor medium used, the trend of flux from various formulations remained identical in a definite receptor composition. A dermal/transdermal selectivity index ( $S$ value) was calculated for evaluation of skin-targeting efficiency. Squarticles showed higher $S$ values than the control, revealing a preferred targeting to the skin reservoir by squarticles based on the in vitro experimental setup in this study. However, caution is needed when comparing $S$ values among different formulations since the receptor composition may affect the measured flux. The application of cyanoacrylate skin-surface casting led to removal of the infundibular drug content in the follicles. Table II shows the recovery of DPCP from the casts. Significantly more DPCP was detected in the follicles by application of squarticles than by $\mathrm{PG}$ dispersion. NE gave rise to a 2.1-fold increase in the drug amount in the follicles compared with the free control.

As shown in Table III, the skin deposition of minoxidil from the PG vehicle is $227 \mu \mathrm{g} / \mathrm{g}$. NLC, and NE could increase the skin uptake to 588 and $324 \mu \mathrm{g} / \mathrm{g}$, respectively. A 2.1-fold increase in minoxidil deposition was accomplished by the NLC type, whereas the deposition of the NE type showed no significant difference $(p>0.05)$ as compared to the control group. Contrary to the case of DPCP, squarticles showed a lower minoxidil flux $(p<0.05)$ with respect to the control solution. The NE type had a 2-fold lower flux compared to the solution. This implies that squarticles could improve minoxidil accumulation in the skin while reducing systemic absorption. Minoxidil loading in NLC and NE greatly increased follicular uptake by 7.0- and 5.2-fold as compared to the free control, suggesting a momentous selectivity for hair follicles. Comparable uptake in follicles $(p>0.05)$ was obtained for the NLC and NE types.

\section{Drug Delivery via Sebum-Removed Skin}

Figure 1 illustrates the skin deposition of the permeants delivered via intact and sebum-removed skin. The difference of DPCP deposition between intact and de-sebumed skin from the free control and NLC was not significant $(p>0.05)$. NE formulation revealed a 2 -fold less DPCP deposition $(p<$ $0.05)$ via sebum-removed skin than intact skin. The result indicates that minoxidil deposition in all formulations was higher $(p<0.05)$ in sebum-removed skin than in intact skin. A 5 -fold increase in skin accumulation was obtained for minoxidil via sebum-removed skin compared to intact skin, while the squarticles (NLC and NE) showed a $\sim 2$-fold increase.

Table II. The In Vitro Skin Deposition at $24 \mathrm{~h}(\mu \mathrm{g} / \mathrm{g})$, flux $\left(\mu \mathrm{g} / \mathrm{cm}^{2} / \mathrm{h}\right)$, and Follicular Uptake $\left(\mu \mathrm{g} / \mathrm{cm}^{2}\right)$ of DPCP Via Nude Mouse Skin Treated With or Without Squarticles

\begin{tabular}{|c|c|c|c|c|c|c|c|}
\hline Type & Skin deposition $(\mu \mathrm{g} / \mathrm{g})$ & $\mathrm{ER}_{\mathrm{SD}}$ & Flux $\left(\mu \mathrm{g} / \mathrm{cm}^{2} / \mathrm{h}\right)$ & $\mathrm{ER}_{\text {flux }}$ & $S$ value & Follicular uptake $\left(\mu \mathrm{g} / \mathrm{cm}^{2}\right)$ & $\mathrm{ER}_{\mathrm{FU}}$ \\
\hline CTL & $209 \pm 50$ & - & $13.2 \pm 0.5$ & - & 15.8 & $1.2 \pm 0.2$ & - \\
\hline NLC & $525 \pm 114 *$ & 2.5 & $15.6 \pm 0.8^{*}$ & 1.2 & 33.8 & $1.7 \pm 0.4^{*}$ & 1.4 \\
\hline $\mathrm{NE}$ & $739 \pm 64 *$ & 3.5 & $28.1 \pm 3.4 *$ & 2.1 & 26.3 & $2.5 \pm 0.4^{*}$ & 2.1 \\
\hline
\end{tabular}

Each value represents the mean and SD $(n=4)$

$* p<0.05$ (the data higher as compared to CTL)

$E R$ enhancement ratio of the flux of squarticle-applied group/the flux of control group, $S$ value the dermal/transdermal selectivity index was calculated as a ratio of skin deposition and the flux value, $C T L$ control, $N L C$ nanostructured lipid carriers, $N E$ nanoemulsions 
Table III. The In Vitro Skin Deposition at $24 \mathrm{~h}(\mu \mathrm{g} / \mathrm{g})$, Flux $\left(\mu \mathrm{g} / \mathrm{cm}^{2} / \mathrm{h}\right)$, and Follicular Uptake $\left(\mu \mathrm{g} / \mathrm{cm}^{2}\right)$ of Minoxidil Via Nude Mouse Skin Treated With or Without Squarticles

\begin{tabular}{lcccccc}
\hline Type & Skin deposition $(\mu \mathrm{g} / \mathrm{g})$ & $\mathrm{ER}_{\mathrm{SD}}$ & Flux $\left(\mu \mathrm{g} / \mathrm{cm}^{2} / \mathrm{h}\right)$ & $\mathrm{ER}_{\mathrm{flux}}$ & $S$ value & Follicular uptake $\left(\mu \mathrm{g} / \mathrm{cm}^{2}\right)$ \\
\hline CTL & $277 \pm 46$ & - & $88.8 \pm 16.7$ & - & 3.1 & $0.8 \pm 0.1$ \\
NLC & $589 \pm 64^{*}$ & 2.1 & $59.1 \pm 15.0$ & 0.7 & 10.0 & $5.4 \pm 0.6^{*}$ \\
NE & $324 \pm 65$ & 1.2 & $44.5 \pm 11.3^{* *}$ & 0.5 & 7.3 & 7.0 \\
\hline
\end{tabular}

Each value represents the mean and $\mathrm{SD}(n=4)$

$* p<0.05$ (the data higher as compared to CTL); **p<0.05 (the data lower as compared to CTL)

$E R$ enhancement ratio of the flux of squarticle-applied group/the flux of control group, $S$ value the dermal/transdermal selectivity index was calculated as a ratio of skin deposition and the flux value, $C T L$ control, $N L C$ nanostructured lipid carriers, $N E$ nanoemulsions

\section{Drug Release from Squarticles}

Figure 2 demonstrates the difference in the release rate of DPCP and minoxidil from squarticles and the aqueous control. DPCP suspension exhibited an initial fast release but quickly reached a plateau in the release amount (Fig. 2a). A higher rate with a continuous rise of DPCP release was detected for squarticles because of complete dissolvation in lipid nanocarriers. After an initial burst, the DPCP release rate reduced but persisted for up to $24 \mathrm{~h}$. We calculated the initial burst effect by the percentage of cumulative amount in receptor at the first $8 \mathrm{~h}$ compared to the cumulative amount at the end of release experiment $(24 \mathrm{~h})$. The burst effect of DPCP between NLC and NE was comparable, since both types showed a $\sim 67 \%$ DPCP release at the initial $8 \mathrm{~h}$ compared to the released amount at $24 \mathrm{~h}$. The NE type showed a release rate considerably greater $(p<0.05)$ than the NLC type. As shown in Fig. 2b, the minoxidil control group also revealed a low release due to limited solubility in aqueous dispersion. Contrary to the case of DPCP, minoxidil in NE showed a comparable release as compared to the free control. The highest minoxidil release was obtained from the NLC type. There was no increase in the released minoxidil amount from squarticles after a 12-h application. All minoxidil formulations (control solution, NLC, and NE) showed a burst release at the first $8 \mathrm{~h}$ since a $87-88 \%$ minoxidil release at the initial $8 \mathrm{~h}$ was calculated as compared to the released amount at $24 \mathrm{~h}$. The burst effect was approximate for the three vehicles.

\section{VEGF Amount of Dermal Papilla Cells}

The presence of VEGF in hair bulbs is necessary for inducing sufficient angiogenesis in hair cycles. The effect of minoxidil on VEGF expression in extracellular and intracellular regions of dermal papilla cells was investigated. Minoxidil in all formulations produced an elevation $(p<$ 0.05) of this protein compared to the non-treatment group as shown in Fig. 3. Cell supernatant and extract exhibited a comparable $(p>0.05)$ VEGF level. The greatest increase was observed by the squarticles in the NLC type $(p<0.05)$, which showed a 2.3-fold increase as compared to the group without minoxidil treatment. No significant difference in VEGF production was found between free minoxidil and NE.

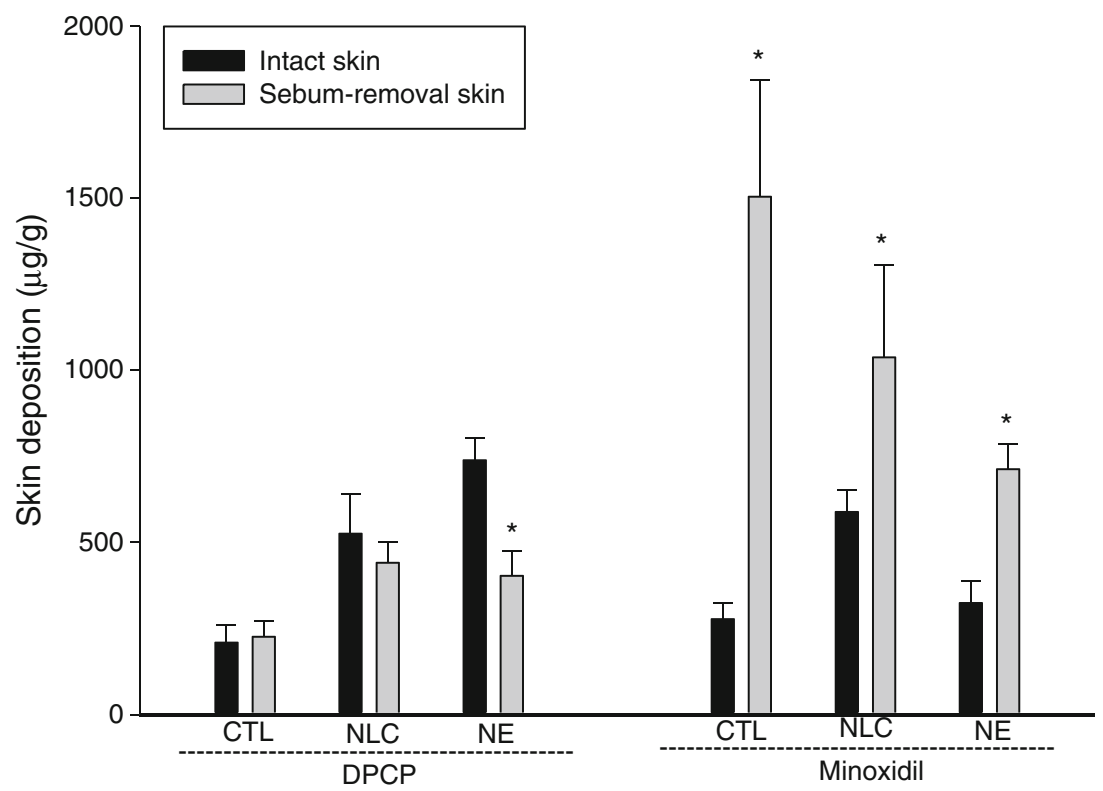

Fig. 1. Comparison of the skin deposition $(\mu \mathrm{g} / \mathrm{g})$ of DPCP and minoxidil in intact skin and sebum-removal skin after in vitro application by aqueous control and squarticles. $* p<0.05$ compared to intact skin. All data represent the mean $\pm \mathrm{SD}$ of four experiments 
a

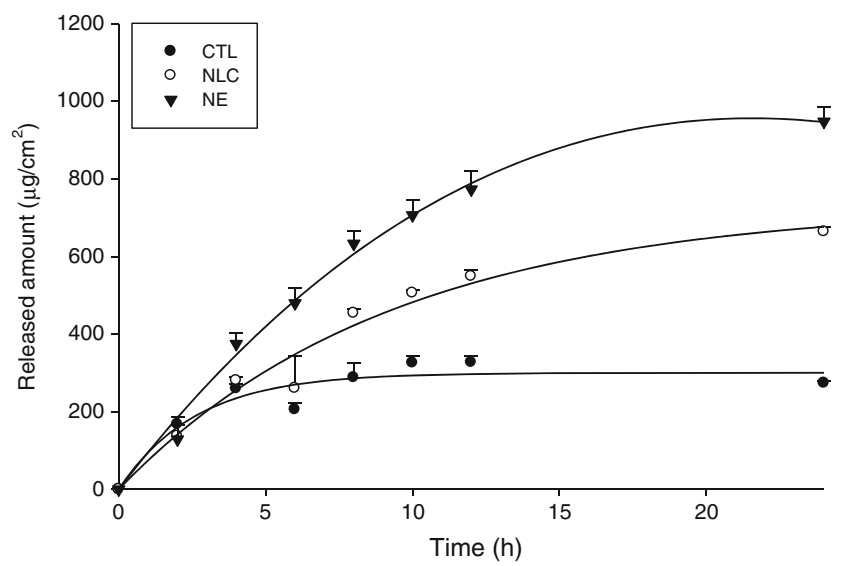

b

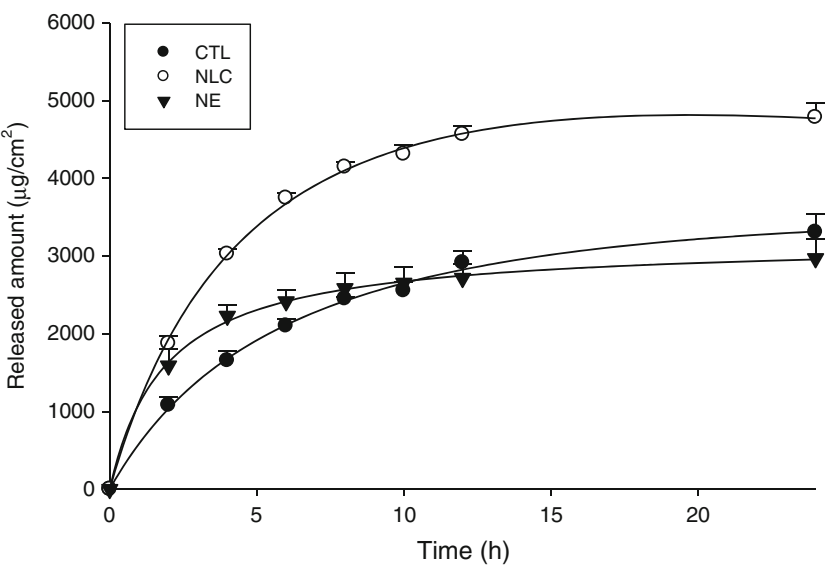

Fig. 2. Released amount versus time profiles of DPCP (a) and minoxidil (b) across cellulose membrane (Cellu-Sep® T1 with a molecular weight cutoff of 3,500) after in vitro application by aqueous control and squarticles. All data represent the mean \pm SD of four experiments

\section{Vertical Observation of Skin by Fluorescence Microscopy}

In order to explore the skin and follicular targeting of squarticles, nanoparticulate distribution in the skin was studied using fluorescence microscopy. Nile red, a lipophilic dye, was incorporated into the lipid matrix for observing fluorescence. Figure 4 depicts the representative fluorescent and $\mathrm{H} \& \mathrm{E}$ images obtained after in vivo application of the aqueous control and squarticles for $6 \mathrm{~h}$. According to the vertical skin section treated with the aqueous control (Fig. 4a), the fluorescence is detected only in the outermost skin surface and a weak signal is observed from the hair follicles. As shown in Fig. 4b and c, it can be observed that the intensity of red fluorescence was stronger from the squarticles than from the control. The red fluorescence was found in the whole skin after treatment of the squarticles. After $6 \mathrm{~h}$ of administration, the squarticles were mainly diffused to the skin surface and the hair follicles where substantial fluorescence was observed. Absorption of the squarticles seemed to follow two routes along the SC and follicles. Squarticles delivered Nile red reached a deeper skin

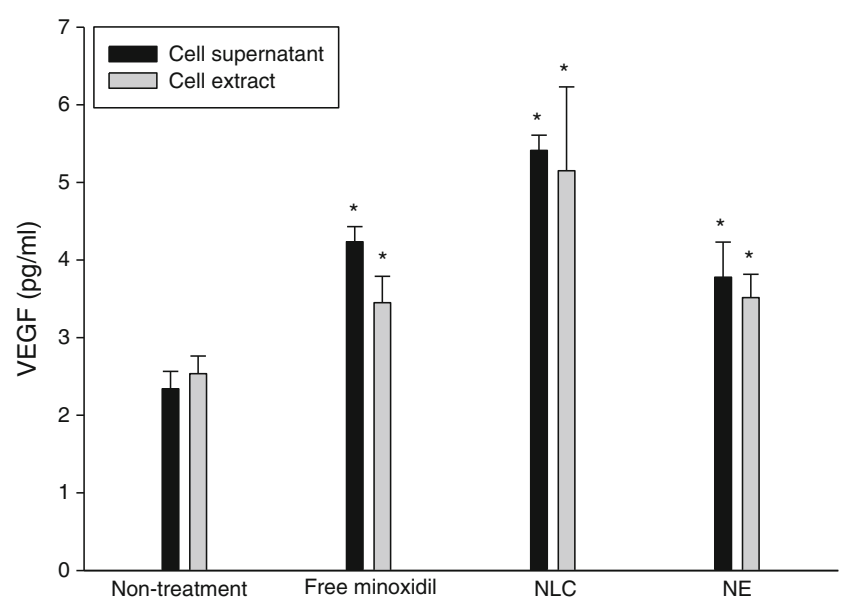

Fig. 3. VEGF expression in supernatant and extract of human dermal papilla cells after incubation with minoxidil $(0.01 \mathrm{M})$ in aqueous control and squarticles. All data represent the mean \pm SD of six experiments stratum as compared to the free control. No significant difference of fluorescence intensity was detected between the NLC type and the NE type.

\section{Horizontal Observation of Skin by CLSM}

In addition to the observation of the vertical skin section, the horizontal expression of the skin examined by CLSM was also performed. Figure 5 shows confocal images from the skin, illustrating collective emissions scanned at $5-\mu \mathrm{m}$ increments from the skin surface. As shown in Fig. 5a, no fluorescence is observed for the skin sample without any treatment. Nile red distribution was greatly affected by the carriers used. Figure 5b is a representative CLSM image of skin treated with the free control, exhibiting localization of Nile red in the vicinity of follicles and hair shafts. For squarticles (Fig. 5c and d), a higher red intensity was obtained compared with the control. Comparing the skin imaging between NLC and NE, a higher intensity of Nile red was seen for NE-treated skin. The red fluorescence distribution was relatively homogeneous over the skin treated by Nile redcontaining NE. Squarticles in the form of NE may permeate into both the SC and follicular ducts.

\section{Viability of Dermal Papilla Cells}

To determine the possible toxicity of squarticles on the skin, we first evaluated papilla cell viability in vitro. Figure 6 shows the survival rate of dermal papilla cells in the presence of an increasing concentration of squarticles. The NE type showed cell viability close to $100 \%$ for all concentrations tested. This indicates that the employed concentrations of NE were nontoxic to dermal papilla cells. At the highest concentration, the NLC type showed a significantly lower $(p<0.05)$ viability percentage of $76 \%$. Treatment with NLC at lower concentrations did not reduce the viability $(p>0.05)$.

\section{In Vivo Skin Irritation Test}

TEWL, erythema, and skin $\mathrm{pH}$ value were recorded to evaluate possible irritation of squarticles in vivo as shown in 


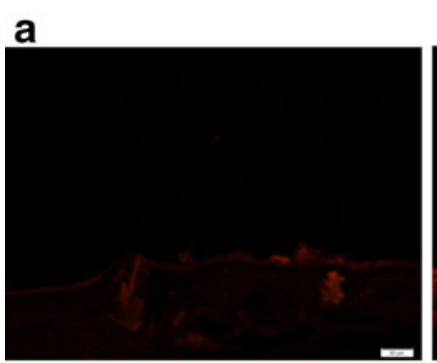

\section{b}

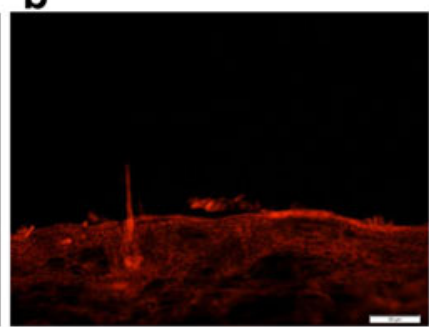

C

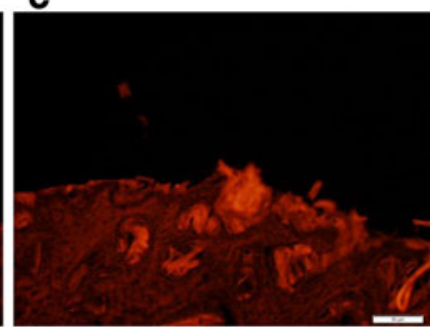

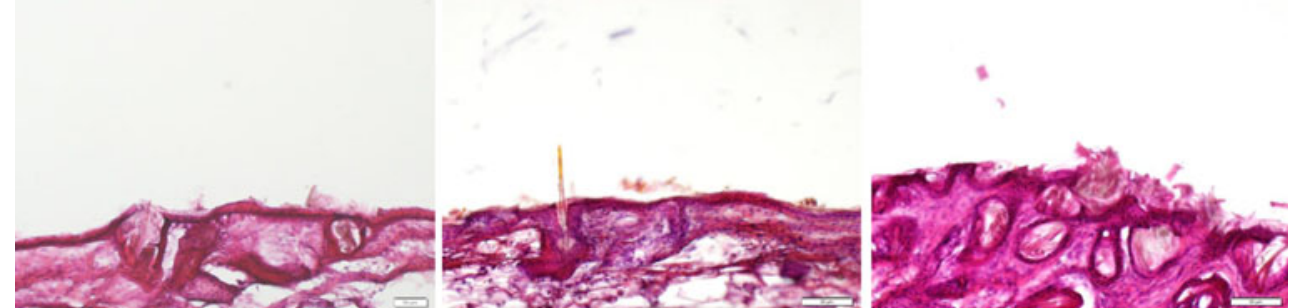

Fig. 4. Fluorescence microscopic images of nude mouse skin with in vivo topical administration of aqueous control (a), squarticles in NLC type (b), and squarticles in NE type (c) containing Nile red (0.1\%) as a dye for $6 \mathrm{~h}$. The upper panel is the fluorescence image detected at 546 and $590 \mathrm{~nm}$ for excitation and emission; the lower panel is the bright-field view of H\&E-stained images

Fig. 7. Values called $\Delta$ values (the level of the treated site minus the level of an adjacent untreated site) were calculated as the results. No significant sensitization resulted if the standard deviation (SD) bar approximated the zero line. As

\section{a}

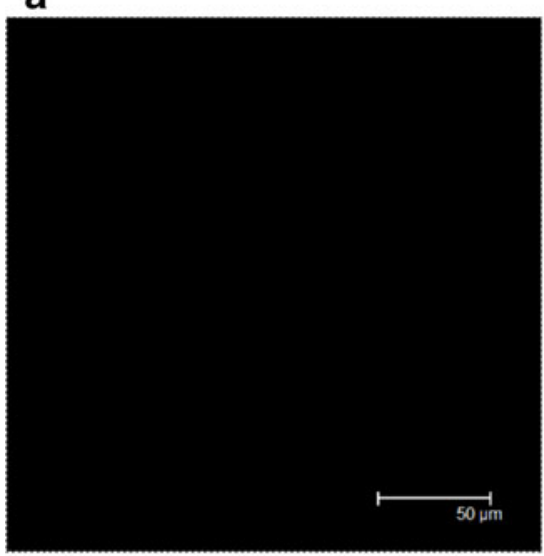

c

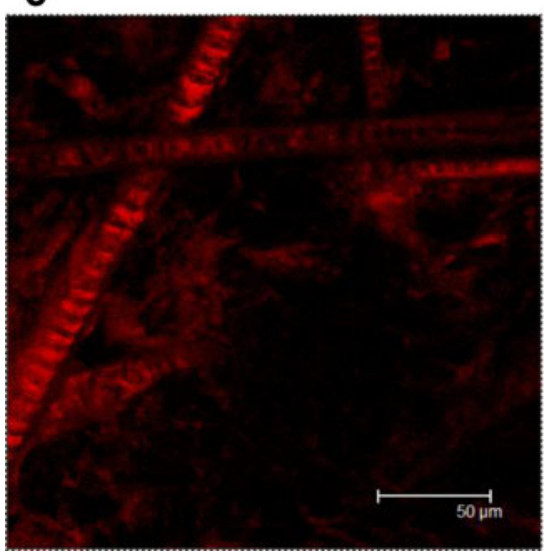

b

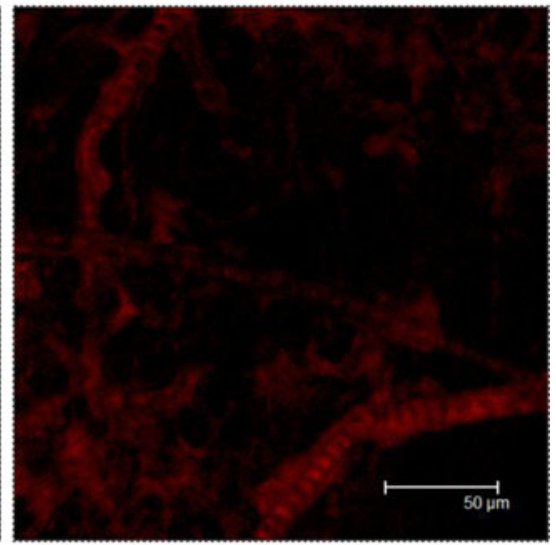

d

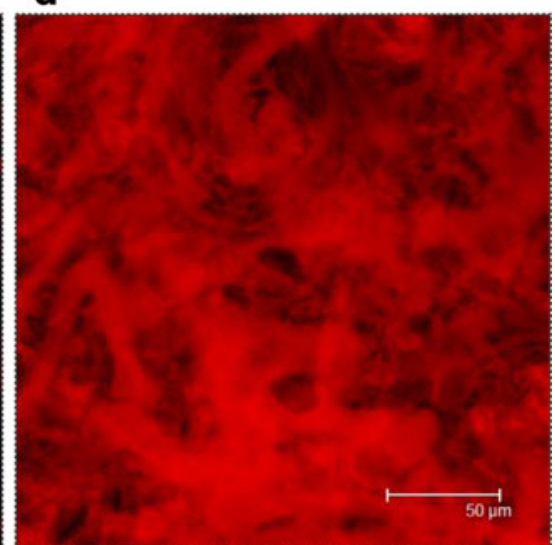

Fig. 5. Confocal micrographs of nude mouse skin at an original magnification of $\times 630$ with non-treatment (blank) (a), in vivo topical administration of aqueous control (b), squarticles in NLC type (c), and squarticles in NE type (d) containing Nile red $(0.1 \%)$ as a dye for $6 \mathrm{~h}$. The image is a summary of 15 fragments at various skin depths 


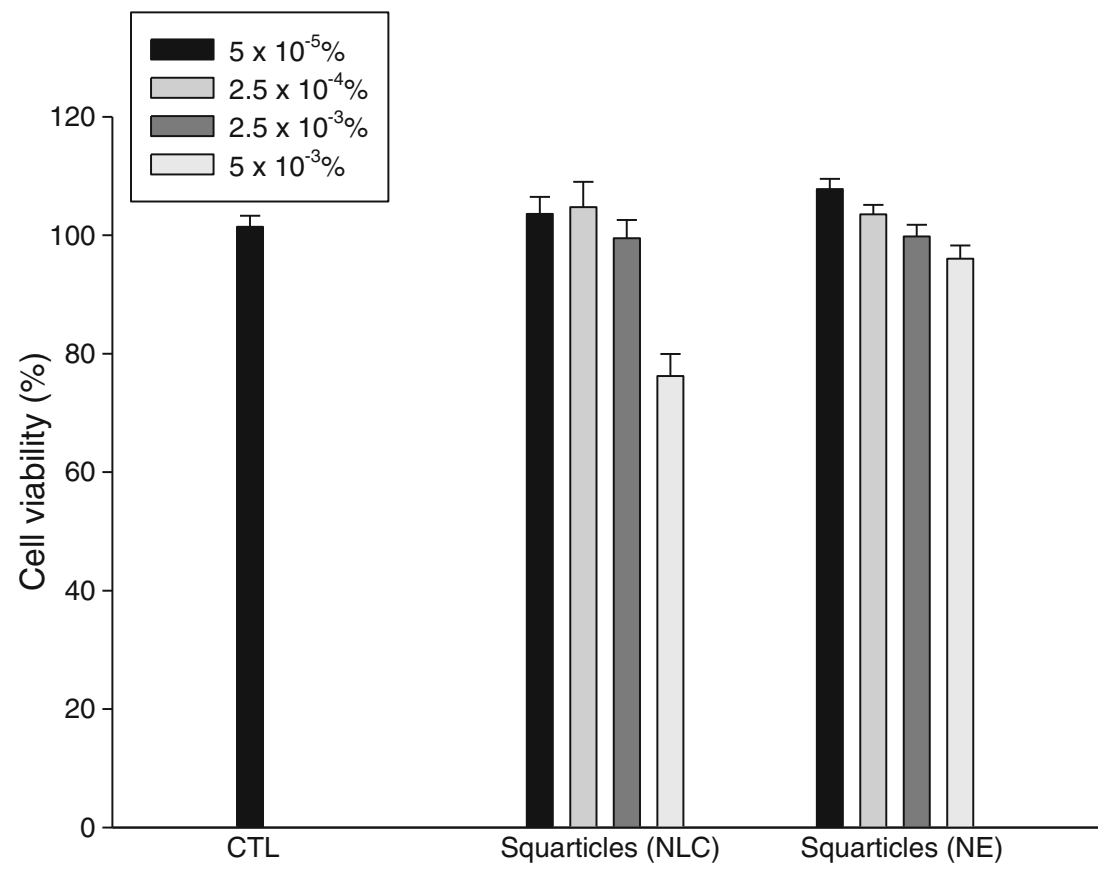

Fig. 6. Viability of human dermal papilla cells after incubation with aqueous control and squarticles at different concentrations. All data represent the mean \pm SD of six experiments

observed in Fig. 7a, TEWL values are increased by all formulations after a 7-day consecutive administration. This may be due to the presence of water in all formulations for hydrating the SC. There was no significant difference $(p>$ $0.05)$ between $\triangle$ TEWL of the squarticles and the aqueous control. Because of the approximation of SD bars to the zero line (Fig. 7b), no sign of erythema is detected for the squarticles. The same result was demonstrated for skin $\mathrm{pH}$, suggesting an acceptable tolerance of squarticles for topical application.

\section{DISCUSSION}

Acetone is commonly employed as a DPCP vehicle in a clinical setting (18). Most commercial products for minoxidil consist of PG, ethanol, and water (19). Novel formulations free of organic solvents are urgently necessary to attenuate skin irritation and optimize pharmacological efficacy. The present work focused on the development of squarticles as lipid nanocarriers for treating hair loss without organic solvents. These nanosystems showed a vital improvement in skin absorption and follicular uptake of encapsulated drugs. Squarticles even reduced possible minoxidil penetration to the receptor of the Franz cell, thus promoting the targeted ability to the skin. A skin-tolerance test demonstrated a negligible irritation of squarticles. Squalene and Precirol were the main materials constituting a lipid core of squarticles. The reason for selecting these lipids was that squalene and glycerides are predominant components of sebum. Precirol is a mixture of mono-, di-, and triglycerides of palmitic and stearic acids.

Squarticles in the NLC type showed a smaller size than the NE type. Precirol, composed of fatty esters, possessed amphiphilic properties (20). The surface-active esters could assist in the emulsification process for reducing particle size and creating a rigid interface. The negative zeta potential of squarticles resulted from negatively charged phospholipids in SPC. According to the previous literature (21), a zeta potential of $>160 \mid \mathrm{mV}$ is demanded for excellent physical stability and of $>|30| \mathrm{mV}$ for good stability. Squarticles showed a surface charge of near $-60 \mathrm{mV}$, which demonstrated a promising ability for maintaining storage stability. PF68 in a

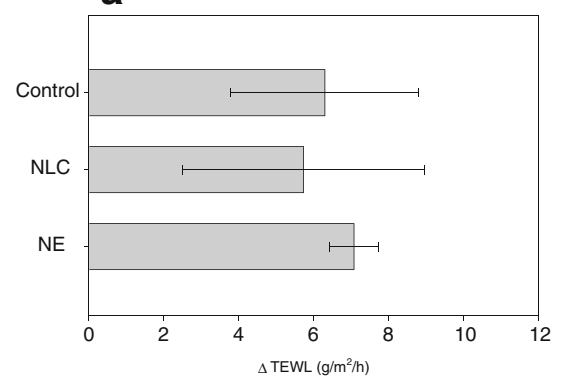

b

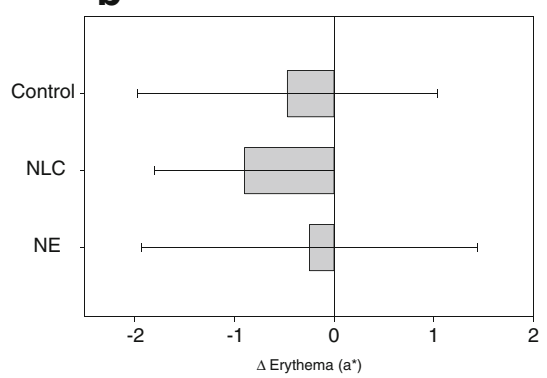

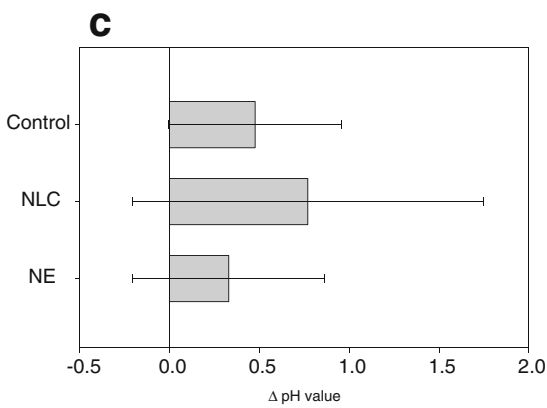

Fig. 7. In vivo skin irritation examination determined by $\Delta$ transepidermal water loss $(\Delta T E W L ; \mathbf{a}), \Delta$ erythema $\left(\Delta \mathrm{a}^{*} ; \mathbf{b}\right)$, and $\Delta$ skin $\mathrm{pH}(\mathbf{c})$ after a 7-day application of aqueous control and squarticles. All data represent the mean \pm SD of six experiments 
the interface also provided a steric stabilization for preventing aggregation. DPCP is a lipophilic molecule with a partition coefficient $(\log P)$ of 3.8. It was expected to easily load into the lipid phase of squarticles. An encapsulation percentage of $\sim 80 \%$ could be achieved for DPCP. Minoxidil is basically regarded as a hydrophilic agent with a $\log P$ of 1.2 (22). The loading capacity of $>60 \%$ for minoxidil was surprising. Mura et al. (23) suggest that minoxidil can be largely incorporated in phospholipid bilayers of liposomes. SPC in the interface of squarticles left spaces to accommodate minoxidil molecules.

The nude mouse was selected as the animal model for the skin-delivery study. It is an acceptable model for human skin in terms of having a similar SC morphology (24). Although nude mouse skin is thinner and more permeable as compared to human skin, it is still an alternative for skindelivery examination due to limited variability among individuals (25). Patients with alopecia areata or androgenetic alopecia often reveal lesional scalp skin with a reduced epidermal barrier function $(26,27)$. Since DPCP and minoxidil are most frequently applied for scalp treatment, nude mouse skin is especially suitable as the alternative for lesional scalp skin because the permeability of the scalp is higher than that of the other anatomical regions (28).

An impressive result of in vitro skin delivery is that, based on the estimated $S$ values obtained under the conditions studied, squarticles significantly lessened the absorbed minoxidil percentage in the receptor and enhanced skin deposition compared to the control dispersion. Similar to the commercial products, PGrich medium was employed as the control vehicle of minoxidil. PG not only solubilized minoxidil, but it also increased the flux across the skin. According to previous studies $(29,30)$, the PG-rich medium could act as penetration enhancers to increase minoxidil receptor penetration. It is reported that minoxidil absorption into systemic circulation leads to unwanted cardiovascular effects $(8,31)$. Drug targeting to specific areas can improve the use of the active agents prone to cause undesired effects. It was advantageous that squarticles resided in the skin strata. Systemic uptake could be excluded, which is beneficial for topically applied minoxidil. A similar result was observed in the case of DPCP. Although both skin deposition and flux were increased by loading DPCP in the squarticles, the $S$ value was increased by loading DPCP into the squarticles. Another successful result was the follicular targeting by squarticles, especially for minoxidil. Hair follicles and bulbs have been regarded as the site of action for hair loss treatment. The selectivity of follicular uptake by the lipid nanocarriers can efficiently deliver the drugs to the target sites. Both systemic side effects and skin irritation may be minimized by this nanoparticle approach.

The nano-sizes and lipidic nature of the lipid nanoparticles assure a close contact to the skin surface with rich sebum (32). According to the encapsulation percentage, $80 \%$ DPCP and $60 \%$ minoxidil were located in about $10 \%$ (lipid matrix) of the volume of the nanosystems. Hence, squarticles would provide a high drug-concentration gradient toward the skin. Though the intact particles permeate into SC with difficulty, the uptake of the lipid components by the skin is possible by the exchange with SC lipids $(33,34)$. The barrier function of $\mathrm{SC}$ could be compromised by this exchange. This interaction between lipid nanoparticles and SC lipids resulted in a decreased penetration into the receptor compartment and the formation of the drug reservoir in the skin. The microscopic images of CLSM demonstrated greater accumulation of squarticles in the skin reservoir than in that of the free control.

Sebum is composed of a lipid mixture, which mixes with SC lipids to form a lipid film on superficial SC (14). It is important in playing a role of drug partitioning from the vehicle to SC. Sebumderived lipids are especially abundant on the scalp skin (26). Sebum removal did not change DPCP deposition from the free control, suggesting that the outermost sebum partitioning was not a rate-limiting process for this drug. On the other hand, skin deposition of minoxidil from the free control could be largely increased by sebum removal. This result suggests that SC partitioning was still a vital barrier retarding minoxidil absorption. DPCP deposition from NE was significantly decreased by sebum removal. The enhancement level of minoxidil deposition after removing the sebum was reduced by loading into the squarticles. These results illustrate the superiority of lipid interaction for increasing drug absorption by squarticles.

The ordered lipid structure inside nanoparticles such as SLN and NLC forms a thin film over the skin surface, resulting in occlusion and hydration effects that disrupt the skin structure and enhance drug absorption (35). This phenomenon could not be observed for NE because of its amorphous form in the lipid matrix. No solid lipid existed in the inner phase of $\mathrm{NE}$, contributing to the core structure without crystalline form. This clarifies the reason for the higher skin deposition of minoxidil in the NLC type than in the NE type. However, it cannot explain the case of DPCP, which showed greater deposition from $\mathrm{NE}$ as compared to NLC. The other mechanisms should be elucidated for DPCP. NE displayed a "softer" characteristic compared to nanoparticles with a crystalline lipid core $(11,36)$. The higher mobility and deformability of NE may allow it to easily squeeze into the skin. Another possibility is that the high percentage of squalene in NE could show a high capacity of interaction to sebum. This inference can be confirmed by reduced DPCP deposition from NE after sebum removal. The confocal imaging also demonstrated a higher fluorescence of NE than NLC in skin. It can be concluded that occlusion may be important for minoxidil absorption from squarticles, whereas sebum partitioning is important for skin delivery of DPCPloaded nanoparticles.

With respect to DPCP, the NE type showed a higher release across cellulose membrane than the NLC type. This trend was the same with in vitro skin permeation. DPCP was mainly dispersed in lipid cores. The release could be affected by a certain composition of the lipid matrix. The lipid structure of NE was more imperfect than that of NLC, and the entrapped drug was released more easily (37). It is expected that minoxidil was largely located on the surface of the nanoparticles because of the lower lipophilicity compared to DPCP. The smaller size of the NLC type created a greater total surface area than $\mathrm{NE}$ did, leading to a higher release rate. A lower encapsulation efficiency and location in the particulate shell of minoxidil in squarticles may support the significant burst-release effect. There are two modes for the drug release from nanoparticles in the skin-delivery process: the drug is released from nanoparticles prior to reaching the skin and the nanoparticles form a depot within the skin and appendages where the drug is slowly released (7). Thus the squarticles presented a combination of free drug, released 
drug, and entrapped drug for promoting delivery into the skin.

The existence of squalene and fatty esters in the squarticles increased the drug uptake into the hair follicles. These components easily mixed with follicular sebum, carrying the drug to form a reservoir in the follicles. SPC in the nanoparticulate surface also played an important role for enhancing follicular targeting. Previous studies $(30,31)$ have suggested that liposomes, which are organized by phospholipids, can deposit minoxidil in follicles. That is why minoxidil showed greater follicular uptake enhancement by squarticles than DPCP. The fluorescence imaging of a vertical skin section verified the formation of follicular depot by squarticles. The diameters of follicular orifices exhibit great variations in body sites. According to the survey by Otberg et al. (38), the smallest diameters are detected on the forehead $(66 \mu \mathrm{m})$ and forearm $(78 \mu \mathrm{m})$. The calf region shows the largest orifice diameter of about $170 \mu \mathrm{m}$. According to histology shown in Fig. 4, the diameter of follicles of nude mouse skin is 40$60 \mu \mathrm{m}$. The diameter of transport pathways for human and mouse follicles was greatly larger than the size of our squarticles $(<200 \mathrm{~nm})$. Follicles thus may provide a sufficient space allowing nanoparticle penetration. The optimization of particle size for follicular delivery has been largely investigated. Rolland et al. (39) report that the particle size of $<3 \mu \mathrm{m}$ facilely penetrated into follicles. Toll et al. (40) investigated transfollicular permeation of microspheres at a size of $0.75-6.0 \mu \mathrm{m}$. They demonstrated a maximum penetration depth by an optimal microsphere size of $1.5 \mu \mathrm{m}$. Lademann et al. (41) report on a specific transport mechanism in follicles is described which would favor particle sizes between 300 and $600 \mathrm{~nm}$. Shim et al. (22) studied the permeation of polymeric nanoparticles with 40 and $130 \mathrm{~nm}$ through rodent skin, and found that decreased particle size promotes follicular delivery. Vogt et al. (42) found that particles in the size range of $750 \mathrm{~nm}$ remain in the superficial area of infundibulum, while particles sized $40 \mathrm{~nm}$ penetrate deeper into follicles and also through follicular epithelium. There is a great discrepancy of the optimized particle size for follicular selectivity. Our nanoparticles showed a diameter of $<200 \mathrm{~nm}$. We believed that the particle size is less important for follicular entrance. The penetration rate is more affected by follicular density than follicular orifice diameter $(43,44)$, especially the penetration via scalp skin (45). The follicular epithelium is more permeable than the interfollicular epithelium (46). The high skin deposition created by squarticles may result from the drug delivery via the appendageal route. The scalp has follicles with a very high density. It is especially beneficial for squarticles to exert targeting efficiency.

Dermal papilla cells reside in the base of follicles and are considered fundamental for hair cycling and growth (47). The papilla cells are the principal target site for minoxidil to upregulate VEGF and develop vascularization (48). VEGF is known to increase follicular size, hair growth, and hair thickness. Our results showed that minoxidil in both squarticles and aqueous dispersion increased VEGF expression, with the NLC type revealing the highest amounts. This indicates that NLC may target the papilla cells by cell uptake or membrane fusion. On the other hand, it must be recognized that minoxidil might release from nanoparticles before interacting with cells. The higher minoxidil release rate from NLC than from NE may contribute to greater upregulation of VEGF.
The cytotoxicity assay of squarticles against dermal papilla cells showed viability close to $100 \%$ except for the highest concentration of the NLC type. This result signifies that the squarticles are generally nontoxic to hair bulbs. TEWL is a parameter to reflect SC integrity. A slight increase of TEWL was found for squarticles, which may be due to SC disruption by water. This occurrence was also detected by the aqueous solution. Based on the profiles of erythema and skin $\mathrm{pH}$ value, squarticles demonstrated a negligible irritation to skin. Contact dermatitis is a common adverse event for both topically applied DPCP and minoxidil. Dermatitis can occur in $6 \%$ of patients treated with $5 \%$ minoxidil solution (6). It is possible that the drug can be entrapped into lipid nanoparticles to eliminate skin toxicity by avoiding direct contact $(19,49)$. Squarticles may have the same effect. The efficient targeting to follicles by squarticles also minimizes elicitation of skin irritation. Further study is needed to explore this prospect.

\section{CONCLUSIONS}

The current investigation evaluated the possible targeting of squarticles to skin and hair follicles. DPCP and minoxidil for treating hair loss were utilized as model drugs in this study. The experimental results showed that squarticles could enhance skin deposition of DPCP and minoxidil when compared to the $30 \%$ PG solution. Fluorescence imaging demonstrated that the nanoparticles translocated to a deeper skin strata than the control group. Both sebum fusion and increased release rate were predominant mechanisms governing drug accumulation in the skin by squarticles. Follicular delivery is a promising pathway for alopecia therapy. Squarticles could effectively carry the drugs to the follicles while limiting drug penetration through the skin. Squarticles with minoxidil upregulated VEGF expression of human dermal papilla cells, indicating a targeted efficiency to these cells. Both the NLC type and NE type of squarticles basically suggested an acceptable tolerance on skin. The findings in this study indicate a feasible approach of using squarticles for improving drug accumulation to the skin and follicles. It is anticipated that squarticles can act as a nanocarrier platform for optimization of treatment of follicle-related diseases such as alopecia, folliculitis, rosacea, and acne.

\section{REFERENCES}

1. MacDonald Hull SP, Wood ML, Hutchinson PE, Sladden M, Messenger AG. Guidelines for the management of alopecia areata. Br J Dermatol. 2003;149:692-9.

2. Avgerinou G, Gregoriou S, Rigopoulos D, Stratigos A, Kalogeromitros D, Katsambas A. Alopecia areata: topical immunotherapy treatment with diphencyprone. J Eur Acad Dermatol Venereol. 2008;22:320-3.

3. Messenger AG, Rundegren J. Minoxidil: mechnisms of action on hair growth. Br J Dermatol. 2004;150:186-94.

4. Tsuboi R, Itami S, Inui S, Ueki R, Katsuoka K, Kurata S, et al. Guidelines for the management of androgenetic alopecia (2010). J Dermatol. 2011;38:1-8.

5. Pray WS. Prevention and treatment of hair loss in men. US Pharmasist. 2008;33:16-20.

6. Alkhalifah A, Alsantali A, Wang E, EcElwee KJ, Shapiro J. Alopecia areata update. Part II. Treatment. J Am Acad Dermatol. 2010;62:191-202.

7. Mura S, Manconi M, Sinico C, Valenti D, Fadda AM. Penetration enhancer-containing vesicles (PEVs) as carriers for cutaneous delivery of minoxidil. Int J Pharm. 2009;380:72-9. 
8. Georgala S, Befon A, Maniatopoulou E, Georgala C. Topical use of minoxidil in children and systemic side effects. Dermatology. 2007;214:101-2.

9. Zhao Y, Brown MB, Jones SA. The effects of particle properties on nanoparticle drug retention and release in dynamic minoxidil foams. Int J Pharm. 2010;383:277-84.

10. Baspinar Y, Keck CM, Borchert HH. Development of a positively charged prednicarbate nanoemulsion. Int $\mathrm{J}$ Pharm. 2010;383:201-8.

11. Wen CJ, Yen TC, Al-Suwayeh SA, Chang HW, Fang JY. In vivo real-time fluorescence visualization and brain-targeting mechanisms of lipid nanocarriers with different fatty ester:oil ratios. Nanomedicine. 2011;6:1545-59.

12. Huang ZR, Lin YK, Fang JY. Biological and pharmacological activities of squalene and related compounds: potential uses in cosmetic dermatology. Molecules. 2009;14:540-54.

13. Blume-Peytavi U, Vogt A. Human hair follicle: reservoir function and selective targeting. Br J Dermatol. 2011;165 Suppl 2:13-7.

14. Campbell CSJ, Contreras-Rojas LR, Delgado-Charro MB, Guy RH. Objective assessment of nanoparticle disposition in mammalian skin after topical exposure. J Control Release. 2012;162:201-7.

15. Teichmann A, Jacobi U, Ossadnik M, Richter H, Koch S, Sterry $\mathrm{W}$, et al. Differential stripping: determination of the amount of topically applied substances penetrated into the hair follicles. J Invest Dermatol. 2005;125:264-9.

16. Lachgar S, Charveron M, Gall Y, Bonafe JL. Minoxidil upregulates the expression of vascular endothelial growth factor in human hair dermal papilla cells. Br J Dermatol. 1998;138:40711.

17. Zhang LW, Al-Suwayeh SA, Hsieh PW, Fang JY. A comparison of skin delivery of ferulic acid and its derivatives: evaluation of their efficacy and safety. Int J Pharm. 2010;399:44-51.

18. Gargs S, Messenger AG. Alopecia areata: evidence-based treatments. Semin Cutan Med Surg. 2009;28:15-8.

19. Padois K, Cantiéni C, Bertholle V, Bardel C, Pirot F, Falson F. Solid lipid nanoparticles suspension versus commercial solutions for dermal delivery of minoxidil. Int J Pharm. 2011;416:300-4.

20. Lin CH, Fang YP, Al-Suwayeh SA, Yang SY, Fang JY Percutaneous absorption and antibacterial activities of lipid nanocarriers loaded with dual drugs for acne treatment. Biol Pharm Bull. 2013;36:276-86.

21. Kovacevic A, Savic S, Vuleta G, Müller RH, Keck CM Polyhydroxy surfactants for the formulation of lipid nanoparticles (SLN and NLC): effects on size, physical stability and particle matrix structure. Int J Pharm. 2011;406:163-72.

22. Shim J, Seok SH, Park WS, Han SH, Kim J, Chang IS. Transdermal delivery of minoxidil with block copolymer nanoparticles. J Control Release. 2004;97:477-84.

23. Mura S, Manconi M, Valenti D, Sinico C, Vila AO, Fadda AM. Transcutol containing vesicles for topical delivery of minoxidil. J Drug Target. 2011;19:189-96.

24. Yamashita N, Tachibana K, Ogawa K, Tsujita N, Tomita A. Scanning electron microscopic evaluation of the skin surface after ultrasound exposure. Anat Rec. 1997;247:455-61.

25. Lee WR, Shen SC, Pai MH, Yang HH, Yuan CY, Fang JY. Fractional laser as a tool to enhance the skin permeation of 5aminolevulinic acid with minimal skin disruption: a comparison with conventional erbium: YAG laser. J Control Release. 2010;145:124-33.

26. O'goshi $\mathrm{K}$, Iguchi $\mathrm{M}$, Tagami $\mathrm{H}$. Functional analysis of the stratum corneum of scalp skin: studies in patients with alopecia areata and androgenetic alopecia. Arch Dermatol Res. 2000;292:605-11.

27. Betz RC, Pforr J, Flaquer A, Redler S, Hanneken S, Eigelshoven $\mathrm{S}$, et al. Loss-of-function mutations in the filaggrin gene and alopecia areata: strong risk factor for a severe course of disease in patients comorbid for atopic disease. J Invest Dermatol. 2007;127:2539-43.

28. Feldmann RJ, Maibach HI. Regional variation in percutaneous penetration of ${ }^{14} \mathrm{C}$ cortisol in man. $\mathrm{J}$ Invest Dermatol. 1967;48:181-3.

29. Grice JE, Ciotti S, Weiner N, Lockwood P, Cross SE, Roberts MS. Relative uptake of minoxidil into appendages and stratum corneum and permeation through human skin in vitro. J Pharm Sci. 2010;99:712-8.

30. Kwon TK, Kim JC. In vitro skin permeation of monolein nanoparticles containing hydroxypropyl $\beta$-cyclodextrin/minoxidil complex. Int J Pharm. 2010;392:268-73.

31. Jain B, Singh B, Katare OP, Vyas SP. Development and characterization of minoxidil-loaded liposomal system for delivery to pilosebaceous units. J Liposome Res. 2010;20:105-14.

32. Cirri M, Bragagni M, Mennini N, Mura P. Development of a new delivery system consisting in "drug-in cyclodextrin-in nanostructured lipid carriers" for ketoprofen topical delivery. Eur J Pharm Biopharm. 2012;80:46-53.

33. Lombardi Borgia S, Regehly M, Sivaramakrishnan R, Mehnert W, Korting HC, Danker K, et al. Lipid nanoparticles for skin penetration enhancement-correlation to drug localization within the particle matrix as determined by fluorescence and parelectric spectroscopy. J Control Release. 2005; 110:151-63.

34. Lin YK, Al-Suwayeh SA, Leu YL, Shen FM, Fang JY. Squalenecontaining nanostructured lipid carriers promote percutaneous absorption and hair follicle targeting of diphencyprone for treating alopecia areata. Pharm Res. 2013;30:435-46.

35. Abdel-Mottaleb NMA, Neumann D, Lamprecht A. Lipid nanocapsules for dermal application: a comparative study of lipid-based versus polymer-based nanocarriers. Eur J Pharm Biopharm. 2011;79:36-42.

36. Wang JJ, Liu KS, Sung KC, Tsai CY, Fang JY. Skin permeation of buprenorphine and its ester prodrugs from lipid nanoparticles: lipid emulsion, nanostructured lipid carriers, and solid lipid nanoparticles. J Microencapsul. 2009;26:734-47.

37. Lin YK, Huang ZR, Zhuo RZ, Fang JY. Combination of calcipotriol and methotrexate in nanostructured lipid carriers for topical delivery. Int J Nanomed. 2010;5:117-28.

38. Otberg N, Richter H, Schaefer H, Blume-Peytavi U, Sterry W, Lademann J. Variations of hair follicle size and distribution in different body sites. J Invest Dermatol. 2004;122:14-9.

39. Rolland A, Wagner N, Chatelus A, Shroot B, Schaefer H. Sitespecific drug delivery to pilosebaceous structures using polymeric microspheres. Pharm Res. 1993;10:1738-44.

40. Toll R, Jacobi U, Richter H, Lademann J, Schaefer H, BlumePeytavi U. Penetration profile of microspheres in follicular targeting of terminal hair follicles. J Invest Dermatol. 2004;123:168-76.

41. Lademann J, Weigmann HJ, Rickmeyer C, Barthelmes H, Schaefer H, Müller G, et al. Penetration of titanium dioxide microparticles in a sunscreen formulation into the horny layer and the follicular orifice. Skin Pharmacol Appl Skin Physiol. 1999;12:247-56.

42. Vogt A, Combadiere B, Hadam S, Stieler KM, Lademann J, Schaefer $\mathrm{H}$, et al. $40 \mathrm{~nm}$, but not 750 or $1,500 \mathrm{~nm}$, nanoparticles enter epidermal CD1a+ cells after transcutaneous application on human skin. J Invest Dermatol. 2006;126:1316-22.

43. Tur E, Maibach HI, Guy RH. Percutaneous penetration of methyl nicotinate at three anatomic sites: evidence for an appendageal contribution to transport? Skin Pharmacol. 1991:4:230-4.

44. Wosicka H, Cal K. Targeting to the hair follicles: current status and potential. J Dermatol Sci. 2010;57:83-9.

45. Ogiso T, Shiraki T, Okajima K, Tanino T, Iwaki M, Wada T. Transfollicular drug delivery: penetration of drugs through human scalp skin and comparison of penetration between scalp and abdominal skins in vitro. J Drug Target. 2002;10:369-78.

46. Otberg N, Patzelt A, Rasulev U, Hagemeister T, Linscheid M, Sinkgraven $\mathrm{R}$, et al. The role of hair follicles in the percutaneous absorption of caffeine. Br J Clin Pharmacol. 2007;65:488-92.

47. Driskell RR, Clavel C, Rendl M, Watt FM. Hair follicle dermal papilla cells at a glance. J Cell Sci. 2011;124:1179-82.

48. Han JH, Kwon OS, Chung JH, Cho KH, Eun HC, Kim KH. Effect of minoxidil on proliferation and apoptosis in dermal papilla cells of human hair follicle. J Dermatol Sci. 2004;34:91-8.

49. Castro GA, Coelho ALLR, Oliveira CA, Machecha GAB, Oréfice RL, Ferreira LAM. Formation of ion pairing as an alternative to improve encapsulation and stability and to reduce skin irritation of retinoic acid loaded in solid lipid nanoparticles. Int J Pharm. 2009;381:77-83. 